\title{
A Systematic Review and Meta-Analysis of Therapeutic Efficacy and Safety of Alirocumab and Evolocumab on Familial Hypercholesterolemia
}

\author{
Xiaoyue Ge, ${ }^{1}$ Tiantian Zhu, ${ }^{2}$ Hao Zeng, ${ }^{1}$ Xin $\mathrm{Yu}^{2}{ }^{2}$ Juan $\mathrm{Li}^{1}{ }^{1}$ Shanshan Xie, ${ }^{1}$ Jinjin Wan, \\ Huiyao Yang, ${ }^{3}$ Keke Huang, ${ }^{2}$ and Weifang Zhang ${ }^{1,4}$ \\ ${ }^{1}$ The Second Affiliated Hospital of Nanchang University, Nanchang 330006, China \\ ${ }^{2}$ Teaching and Research Office of Clinical Pharmacology, China Department of Pharmacy, College of Pharmacy, \\ Xinxiang Medical University, Xinxiang 453003, China \\ ${ }^{3}$ Phase I Clinical Trial Center of Chongqing University Cancer Hospital, Chongqing 400030, China \\ ${ }^{4}$ Medical College of Nanchang University, Nanchang 330031, China
}

Correspondence should be addressed to Weifang Zhang; z_weifang@163.com

Received 28 May 2021; Accepted 11 September 2021; Published 31 October 2021

Academic Editor: Dabin Kuang

Copyright (c) 2021 Xiaoyue Ge et al. This is an open access article distributed under the Creative Commons Attribution License, which permits unrestricted use, distribution, and reproduction in any medium, provided the original work is properly cited.

Objectives. The aim of this study was to provide the first study to systematically analyze the efficacy and safety of PCSK9-mAbs in the treatment of familial hypercholesterolemia (FH). Methods. A computer was used to search the electronic Cochrane Library, PubMed/MEDLINE, and Embase databases for clinical trials using the following search terms: "AMG 145", "evolocumab", "SAR236553/REGN727", "alirocumab", "RG7652", "LY3015014", "RN316/bococizumab", "PCSK9", and "familial hypercholesterolemia" up to November 2020. Study quality was assessed with the Cochrane Collaboration's tool, and publication bias was evaluated by a contour-enhanced funnel plot and the Harbord modification of the Egger test. After obtaining the data, a meta-analysis was performed using $\mathrm{R}$ software, version 4.0.3. Results. A meta-analysis was performed on 7 clinical trials (926 total patients). The results showed that PCSK9-mAbs reduced the LDL-C level by the greatest margin, WMD $-49.14 \%$, 95\% CI: -55.81 to $-42.47 \%$, on $\mathrm{FH}$ versus control groups. PCSK9-mAbs also significantly reduced lipoprotein (a) (Lp (a)), total cholesterol (TC), triglycerides (TG), apolipoprotein-B (Apo-B), and non-high-density lipoprotein cholesterol (non-HDL-C) levels and increased HDL-C and apolipoprotein-A1 (Apo-A1) levels of beneficial lipoproteins. Moreover, no significant difference was found between PCSK9-mAbs treatment and placebo in common adverse events, serious events, and laboratory adverse events. Conclusion. PCSK9-mAbs significantly decreased LDL-C and other lipid levels with satisfactory safety and tolerability in $\mathrm{FH}$ treatment.

\section{Introduction}

Familial hypercholesterolemia (FH) is a common genetic disorder that causes high low-density lipoprotein cholesterol (LDL-C) level from birth, which causes atherosclerotic plaque deposition in the arteries and a markedly increased risk of coronary heart disease (CHD) at a young age [1]. In FH, the most common defect is loss-of-function mutations in $\mathrm{LDL}$ receptor alleles. Other more uncommon causes of $\mathrm{FH}$ are defects in apolipoprotein $\mathrm{B}(\mathrm{ApoB})$ and proprotein convertase subtilisin/kexin type 9 serine (PCSK9) [2]. FH includes homozygous and heterozygous types that have different symptoms, risks, and treatments. The incidence of $\mathrm{FH}$ is approximately 1 in 200-500 individuals and confers a significant risk for premature cardiovascular disease (CVD) [3]. Study has reported that the risk of premature CHD is elevated approximately 20 -fold in young untreated heterozygous $\mathrm{FH}$ men and that homozygous $\mathrm{FH}$ patients typically develop CHD by the second decade of life [4].

Over the past decades, lipid-lowering drugs such as stains, ezetimibe, extended-release niacin formulations, and newer bile acid sequestrants have substantially improved 


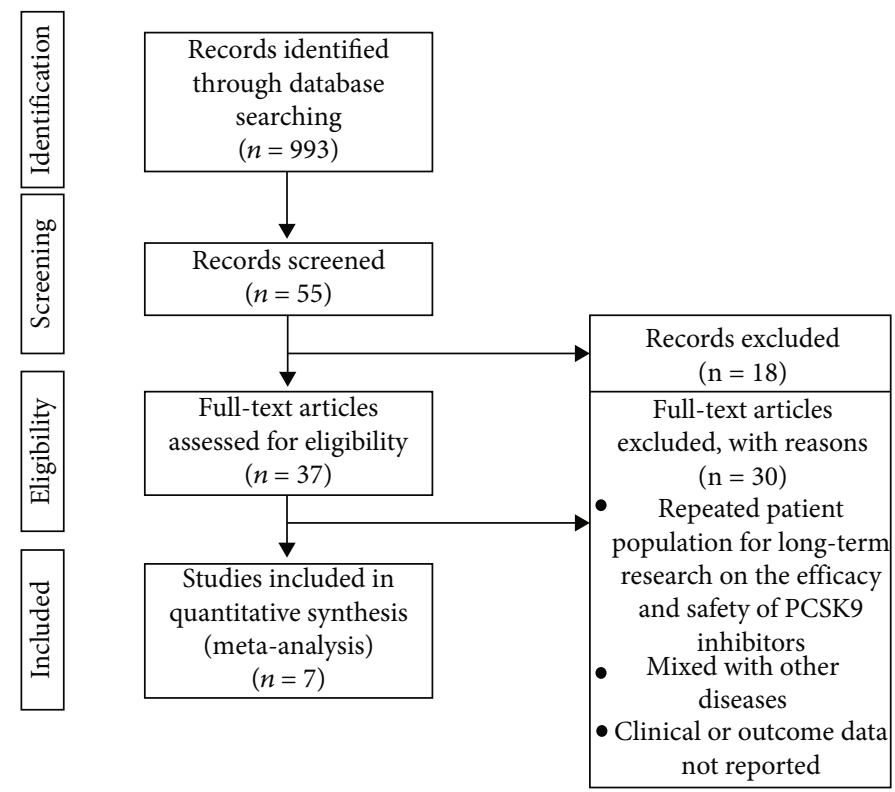

Figure 1: Study and patient selection.

the treatment of $\mathrm{FH}$ patients. However, it has been clinically observed that even if more than $50 \%$ of $\mathrm{FH}$ patients take high-dose statins orally, many patients still do not achieve desirable LDL cholesterol concentrations, and a high risk of CVD remains [5]. PCSK9, a major regulator of $\mathrm{LDL}-\mathrm{C}$ levels, binds to the LDL receptor (LDLR) and is subsequently internalized by the receptor to enhance LDL-C degradation in endo-/lysosomal vesicles in the liver [6]. Phase 1 and 2 trials of PCSK9-mAbs have shown that the level of LDL cholesterol is further reduced by $55-60 \%$ when they are added to existing lipid-lowering treatments, for example, stains alone or statins combined with ezetimibe. Alirocumab/SAR236553/REGN727 and evolocumab/AMG145 are classic human PCSK9-mAbs. In recent years, studies have demonstrated that RG7652 [7], LY3015014 [8], and bococizumab/RN316 [9] are effective for altering the lipidome of plasma and lipoprotein fractions. However, these drugrelated clinical studies were terminated.

Clinical trials have proven that PCSK9-mAbs (alirocumab and evolocumab) decrease the plasma LDL-C level in FH patients. Other lipids and lipoproteins, such as lipoprotein (a) (Lp(a)), total cholesterol (TC), triglycerides (TG), apolipoprotein-B (Apo-B), high-density lipoprotein cholesterol (HDL-C), and apolipoprotein-A1 (Apo-A1), can also benefit [10]. However, no report has comprehensively pinpointed the applicable targets of PCSK9-mAb-FH patients with sufficient clinical outcomes. To confirm the efficacy and safety of PCSK9-mAbs in FH patients, a total of 7 articles (926 patients) were assessed in this meta-analysis.

\section{Methods}

2.1. Literature Search. We followed the methods of our previous study described [11]. In general, we obtained individual participant data from studies identified through systematic searches of the published literature performed using the Cochrane Library, PubMed/MEDLINE, and Embase databases (the following search terms were used: “AMG 145”, “evolocumab”, "SAR236553/REGN727”, “alirocumab”, "RG7652”, “LY3015014”, “RN316/bococizumab”, "PCSK9", and "familial hypercholesterolemia" clinical trial) up to November 2020. We obtained articles in peerreviewed journals for electronic searches. Additional data, especially original data not identified in the electronic databases, were collected from other data resources, and we also performed an additional search of the references of the retrieved studies. Notably, we obtained original data by contacting the corresponding authors when the data were not reported in the identified published articles.

2.2. Selection of Studies for Inclusion in the Review. Cohort studies were included if they met the following criteria: (1) type of study: randomized controlled trials (RCTs); (2) types of participants: FH diagnosis in accordance with clinical criteria or DNA-based analyses; (3) type of interventions: patients received PCSK9-mAbs; and (4) safety and efficacy outcomes of PCSK9-mAbs. The exclusion criteria were as follows: (1) duplicate reports describing the same cohort; (2) certain publication types, such as conference abstracts, letters, comments, case reports, and editorials; (3) repeated patient population for long-term research on the efficacy and safety of PCSK9-mAbs; and (4) non-FH subjects included in the study population.

2.3. Data Extraction. All studies retrieved by the search strategy were independently screened by 2 reviewers (XYG and TTZ). The initial prescreening was performed by reading the titles and abstracts to select relevant studies for further data extraction. Secondary selection was conducted by comprehensively reviewing the full text of all initially identified articles to determine whether the necessary information was reported. Basic information was extracted as follows: 


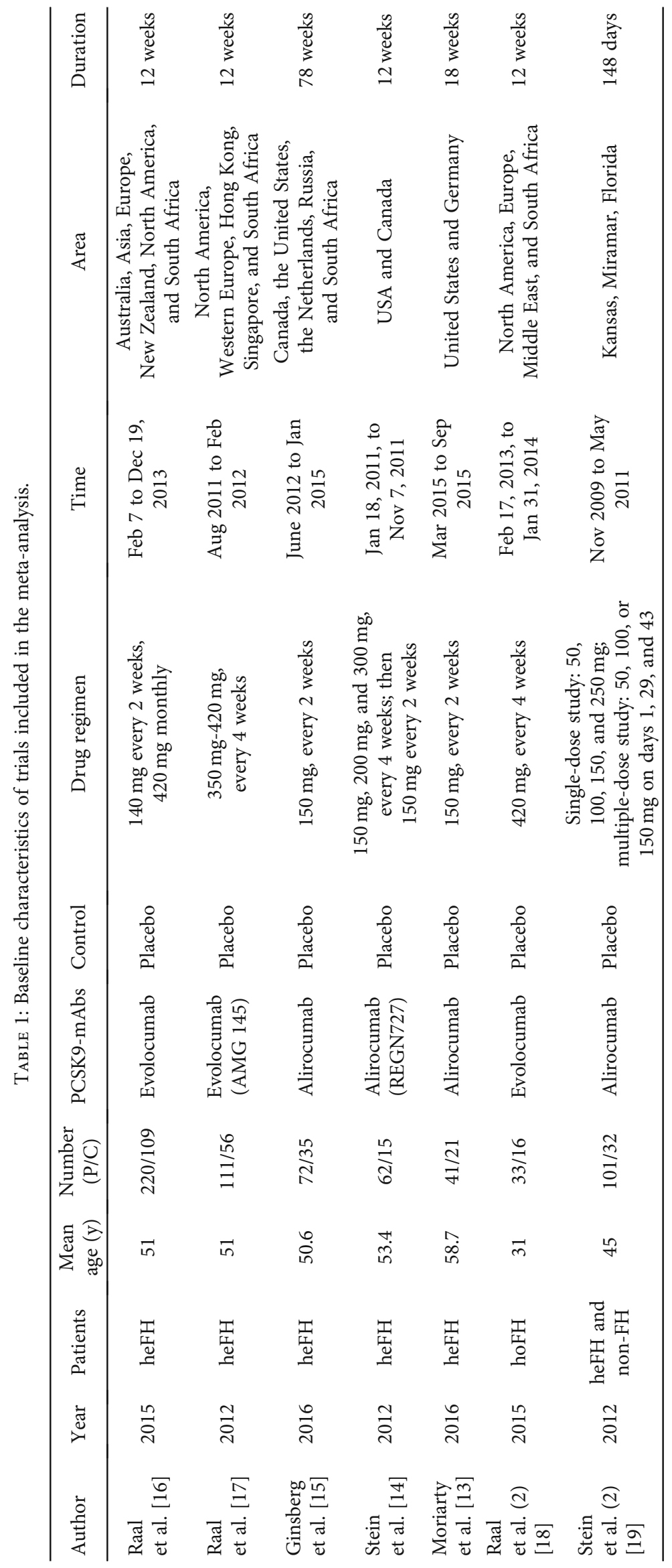




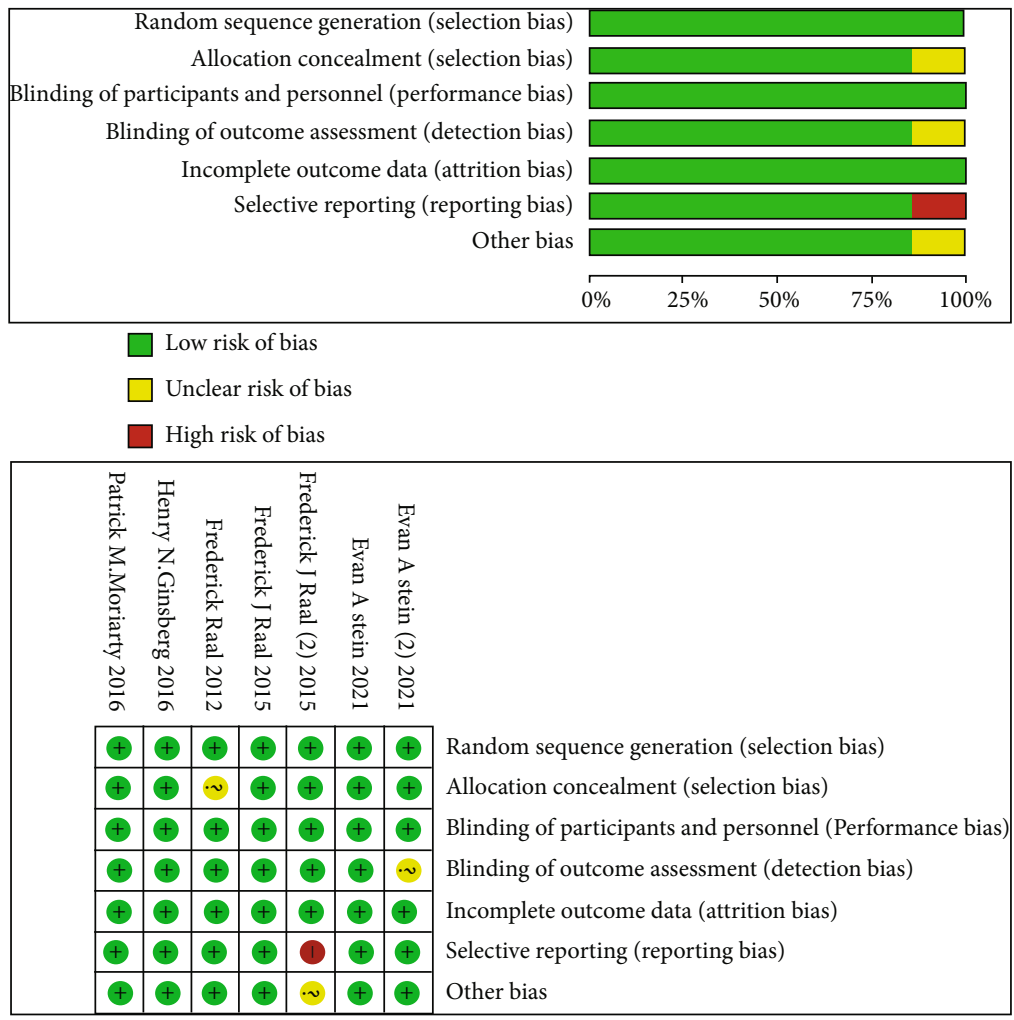

FIGURE 2: Risk-of-bias graph and summary table: review authors' judgments about each risk-of-bias item presented as percentages across all included studies.

author, year, patient number, mean age (y) at baseline, the type of PCSK9-mAbs, control, drug regimen, duration, study time, and area. Then, we extracted the corresponding mean differences, 95\% CI or LS mean percent change, and SE from baseline of each lipid items, including LDL-C, HDL-C, nonHDL-C, TC, Apo-B and Apo-A1, TG, and Lp(a), as the primary outcomes. Safety endpoints covering the common adverse events, serious events, and laboratory adverse events were compared between the treatment and control groups.

2.4. Quality Evaluation. The literature quality evaluation used the Cochrane risk assessment form to conduct risk assessment on the included studies as descripted by "McKenzie et al." [12]. The assessment content includes (1) whether the random method is correct; (2) whether the allocation is hidden; (3) whether the implementer and participants are blinded; (4) whether the result analysis applies the blinding method; whether the data results are fully reported; (5) whether there is selective reporting; and whether there are other biases. According to specific circumstances, the risk assessment results are divided into three situations: high, low, and unclear. High-risk assessment research may lead to unsound analysis results, which are analyzed in sensitivity analysis and subgroup analysis.

\subsection{Appraisal of the Risk of Bias of the Included Studies.} Potential publication bias was evaluated by visually contour-enhanced funnel plots and Egger's test. According to the Egger methods for evaluating publication bias, a two-sided $p$ value of 0.10 or less was regarded as significant.
2.6. Sensitivity Analysis. When substantial heterogeneity was noted between trials, leave-one-out sensitivity analysis was used, which means removing one study each time and repeating the analysis to determine whether exclusion of any one of the included studies altered the results.

2.7. Statistical Analysis. Statistical analyses were performed using $\mathrm{R}$ software, version 4.0.3 ( $\mathrm{R}$ Foundation). The $\chi^{2}$ statistic and independent-samples $T$-tests were used to assess differences in the baseline characteristics of the two groups. The risk ratio (RR) and weighted mean difference (WMD) were calculated and presented with the 95\% confidence interval (CI) for summary estimates. Due to the heterogeneity among the included studies, appropriate statistical models were selected to ensure that the statistical data were estimated correctly. Statistical heterogeneity between studies was assessed using the $\chi^{2}$ test, with a $p$ value of less than 0.1 considered to indicate statistical significance, and heterogeneity was quantified using the inconsistency $\left(I^{2}\right)$ statistic. The $I^{2}$ statistic describes the percentage of total variation across studies due to significant heterogeneity rather than random chance. An $I^{2}$ statistic greater than $50 \%$ suggests considerable heterogeneity among the studies. Publication bias was assessed using contour-enhanced funnel plots. Because the visual interpretation of funnel plot asymmetry is inherently subjective, we also formally tested funnel plot asymmetry using the Harbord modification of Egger's test. Statistical significance was set at a $p$ value $<0.05$. 


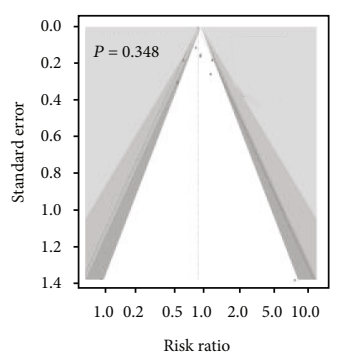

$\square 0.1>\mathrm{P}>0.05$

$\square 0.05>\mathrm{P}>0.01$

$\square \quad \mathrm{P}<0.01$

(a)

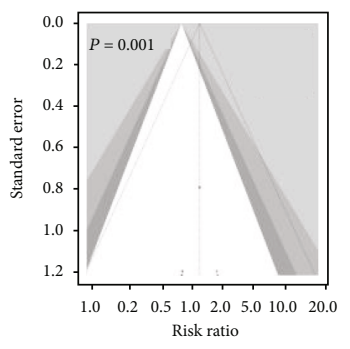

$\square .1>\mathrm{P}>0.05$

$\square 0.05>P>0.01$

$\square \mathrm{P}<0.01$

(c)

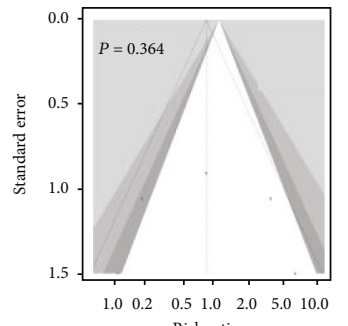

Risk ratio
$0.1>\mathrm{P}>0.05$

$\square \quad 0.05>\mathrm{P}>0.01$

$\square \quad \mathrm{P}<0.01$

(e)

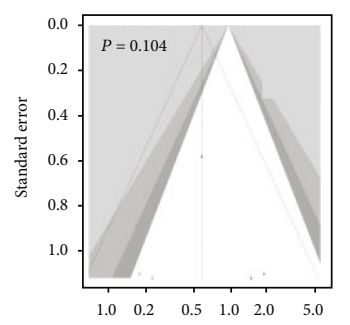

Risk ratio

$\square \quad 0.1>\mathrm{P}>0.05$

$\square \quad 0.05>\mathrm{P}>0.01$

$\square \mathrm{P}<0.01$

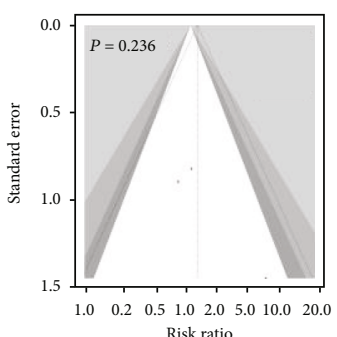

$\square 0.1>\mathrm{P}>0.05$

$\square 0.05>\mathrm{P}>0.01$

$\square \quad \mathrm{P}<0.01$

(b)

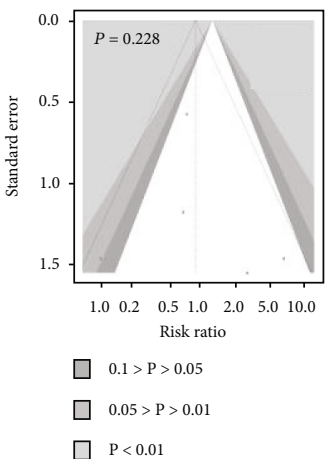

(d)

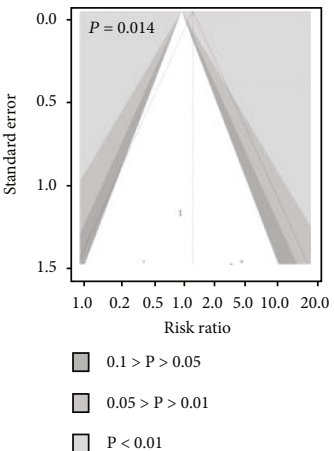

(f)

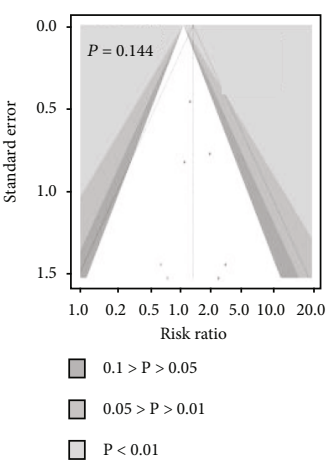

(h)

(g)

Figure 3: Continued. 


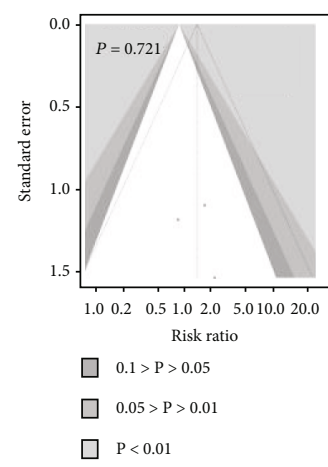

(i)

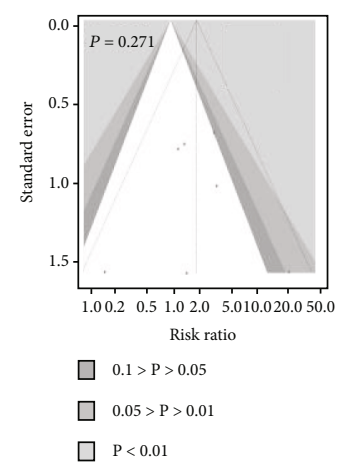

(j)

Figure 3: Appraisal of the risk of bias of the included studies: (a) any adverse events; (b) serious adverse events; (c) leading to treatment discontinuation; (d) adjudicated cardiovascular events; (e) nervous system disorders; (f) creatine kinase (CK $\geq 3 \times \mathrm{ULN})$; (g) headache; (h) nasopharyngitis; (i) abnormal liver function risk (AST/ALT $\geq 3 \times U L N)$; (j) injection site reactions.

\section{Results}

3.1. Study Selection and Characteristics. Literature search results and characteristics were initially obtained from 993 articles, and there were 55 clinical studies. Eighteen papers were removed after reviewing the titles and abstracts. Then, the full text of each of the remaining 37 articles was retrieved for further review to determine whether they met the predetermined criteria. Finally, 7 papers were identified and included in the present study (Figure 1). As a result, 7 studies encompassing a total of 926 patients were selected [13-19]. Among them, 3 trials used evolocumab (AMG 145), and 4 studies used alirocumab (SAR236553/REGN727) treatment. Baseline characteristics were detailed, giving substantially similar basic values between PCSK9$\mathrm{mAbs}$ and controls. The mean age of the subjects ranged from 31 to 59 years old. All trials were published between 2012 and 2016 with a follow-up period ranging from 8 to 78 weeks (Table 1 ) and a low risk of bias (Figure 2).

3.2. Bias Assessment and Consistency Test. Funnel plots were used to investigate the presence of small-study effects and publication bias. Figure 3 shows the contour-enhanced funnel plots and the Harbord modification of the Egger test of the studies included in this meta-analysis for adverse events, such as common adverse events, serious adverse events, and laboratory adverse events. There was no apparent asymmetry for the studies examining PCSK9-mAbs versus placebo for most of the adverse events, other than leading to treatment discontinuation and creatine kinase level $(\mathrm{CK} \geq 3 \times$ upper limit of normal (ULN)).

3.3. Efficacy Outcomes of PCSK9-mAbs. We could see from Figure 4 that PCSK9-mAbs markedly decreased the LDL-C level by $-49.14 \%$, 95\% CI: -55.81 to $-42.47 \%, I^{2}$ : $99 \%$, $p<$ 0.01 (Figure $4(\mathrm{a})$ ), and increased the level of HDL-C by 6.41\%, 95\% CI: 4.09 to $8.73 \%, I^{2}: 95 \%, \quad p<0.01$ (Figure 4(b)), and Apo-A1 by 8.27, 95\% CI: 3.38 to $13.16 \%$, $I^{2}: 99 \%, p<0.01$ (Figure 4(c)). They also decreased the level of Apo-B by $-38.09 \%$, 95\% CI: -45.03 to $31.16 \%, I^{2}$ : $98 \%, p$ $<0.01$ (Figure 4(d)); non-HDL-C by $-46.26 \%$, 95\% CI:
-53.45 to $39.06 \%, I^{2}: 93 \%, p<0.01$ (Figure $4(\mathrm{e})$ ); TC by $-36.47 \%$, 95\% CI: -42.09 to $28.84 \%, I^{2}$ : $97 \%, p<0.01$ (Figure 4(f)); TG by $-10.26 \%, 95 \%$ CI: -18.68 to $-1.84 \%, I^{2}$ : 95\%, $p<0.01$ (Figure 4(g)); and Lp(a) by $-17.65 \%, 95 \% \mathrm{CI}$ : -24.75 to $-10.55 \%, I^{2}: 98 \%, p<0.01$ (Figure $4(\mathrm{~h})$ ).

As a lipid outcome of evolocumab, a significant reduction in LDL-C level was achieved (WMD: $-49.45 \%$, 95\% CI: -57.04 to $-41.85 \%, I^{2}: 99 \%, p<0.01$, Figure $\left.4(\mathrm{a})\right)$. In addition, HDL-C level obviously increased by $5.94 \%$ (95\% CI: 3.11 to $8.76 \%, I^{2}: 97 \%, p<0.01$, Figure $\left.4(\mathrm{~b})\right)$, and ApoA1 level increased by $5.20 \%$ (95\% CI: -1.66 to $12.06 \%, I^{2}$ : $100 \%, p<0.01$, Figure 4(c)). Furthermore, Apo-B level obviously decreased by $-40.12 \%$ (95\% CI: -46.47 to $-33.78 \%, I^{2}$ : $99 \%, p<0.01$, Figure 4(d)), non-HDL-C level by $-54.21 \%$ (95\% CI: -55.48 to $-52.94 \%, \quad I^{2}: 76 \%, \quad p=0.043$, Figure 4(e)), TC level by $-40.30 \%$ (95\% CI: -41.08 to $39.52 \%, I^{2}: 97 \%, p<0.01$, Figure $\left.4(\mathrm{f})\right)$, TG level by $-14.07 \%$ (95\% CI: -19.74 to $-8.41 \%, I^{2}: 97 \%, p<0.01$, Figure $4(\mathrm{~g})$ ), and $\mathrm{Lp}$ (a) level by $-22.56 \%$ (95\% CI: -30.33 to $-14.78 \%, I^{2}$ : $99 \%, p<0.01$, Figure $4(\mathrm{~h}))$ versus placebo.

As a lipid outcome of alirocumab, a significant reduction in LDL-C level was achieved (mean reduction: $-49.10 \%$, 95\% CI: -57.91 to $-40.28 \%, I^{2}: 97 \%, p<0.01$, Figure $4(\mathrm{a})$ ). In addition, HDL-C level obviously increased by $7.12 \%$ (95\% CI: 2.83 to $11.42 \%, I^{2}: 93 \%, p<0.01$, Figure $4(\mathrm{~b})$ ) and Apo-A1 level increased by $11.43 \%$ (95\% CI: 6.19 to 16.66\%, $I^{2}: 93 \%, p<0.01$, Figure 4(c)). Moreover, Apo-B level obviously decreased by $-36.38 \%$ (95\% CI: -43.75 to $-29.01 \%, I^{2}$ : 97\%, $p<0.01$, Figure 4(d)), non-HDL-C level by $-40.79 \%$ (95\% CI: -47.00 to $-34.58 \%, I^{2}$ : $95 \%, p<0.01$, Figure 4(e)), TC level by $-33.80 \%$ (95\% CI: -40.24 to $27.36 \%, I^{2}: 97 \%, p<0.01$, Figure $\left.4(\mathrm{f})\right)$, TG level by $-5.68 \%$ (95\% CI: -5.93 to $-5.43 \%, I^{2}: 0 \%, p=0.968$, Figure $4(\mathrm{~g})$ ), and $\mathrm{Lp}$ (a) level by $-12.89 \%$ (95\% CI: -20.17 to $-5.61 \%, I^{2}$ : $94 \%, p<0.01$, Figure $4(\mathrm{~h}))$ versus placebo.

3.4. Safety Outcomes of PCSK9-mAbs. We compared the safety endpoints covering the common adverse events, serious events, and laboratory adverse events between the PCSK9-mAbs and control groups and found that the overall 


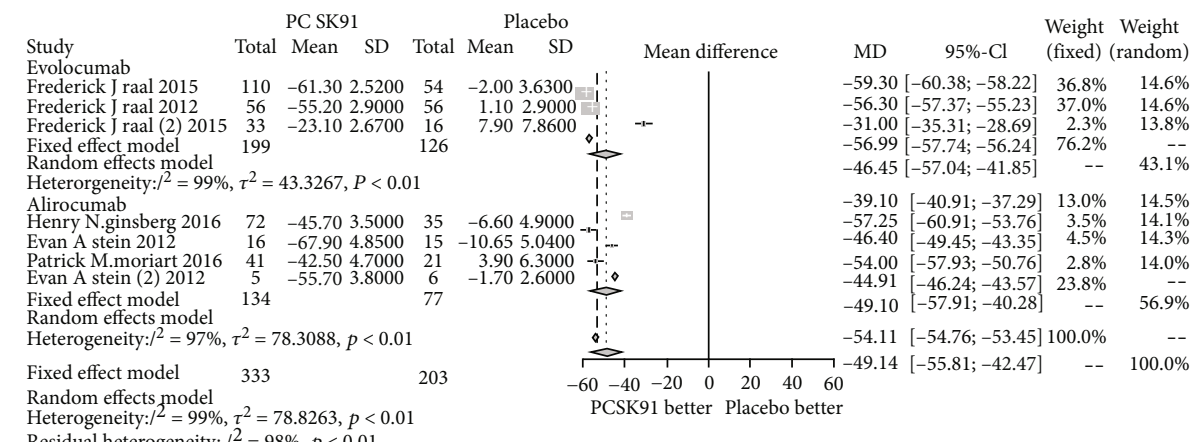

(a)

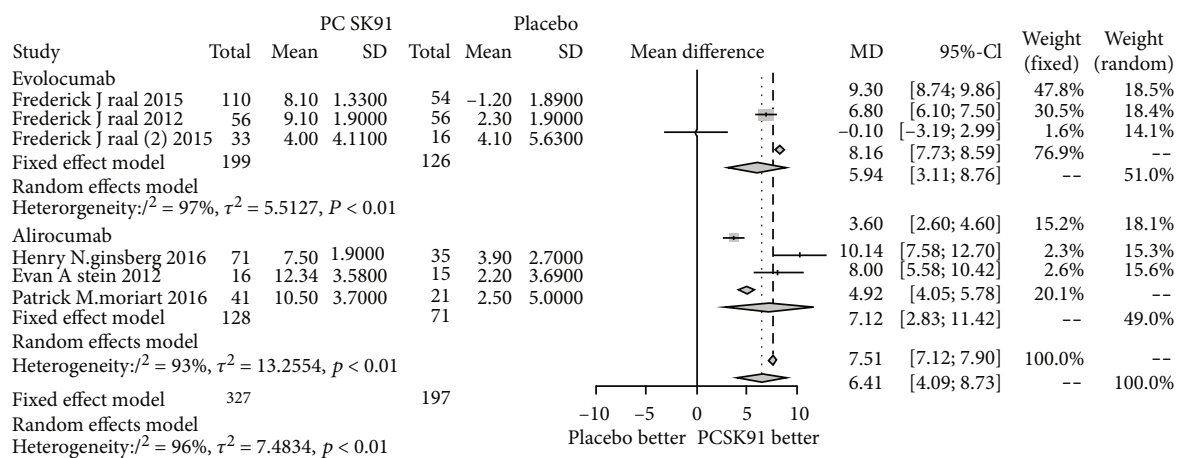

Residual heterogeneity: $/^{2}=95 \%, p<0.01$

(b)

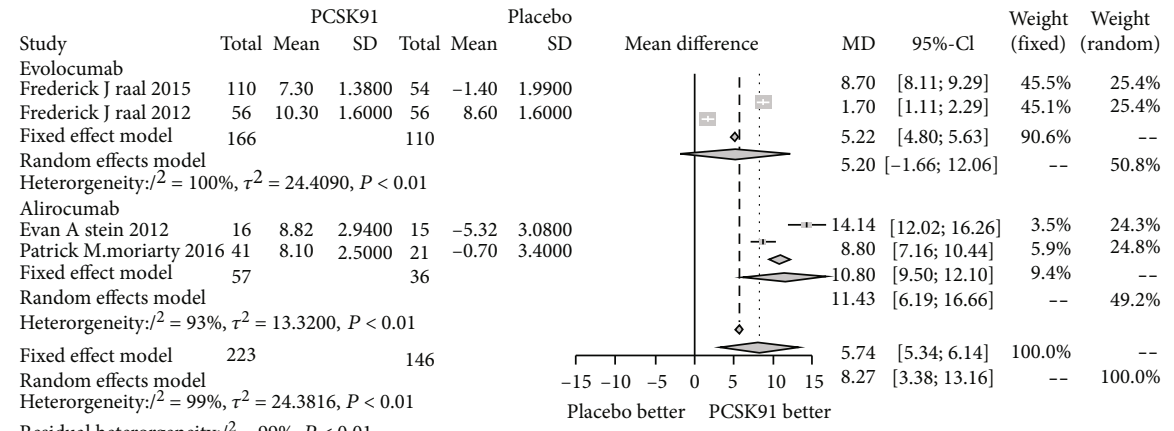

Residual heterorgeneity: $/^{2}=99 \%, P<0.01$

(c)

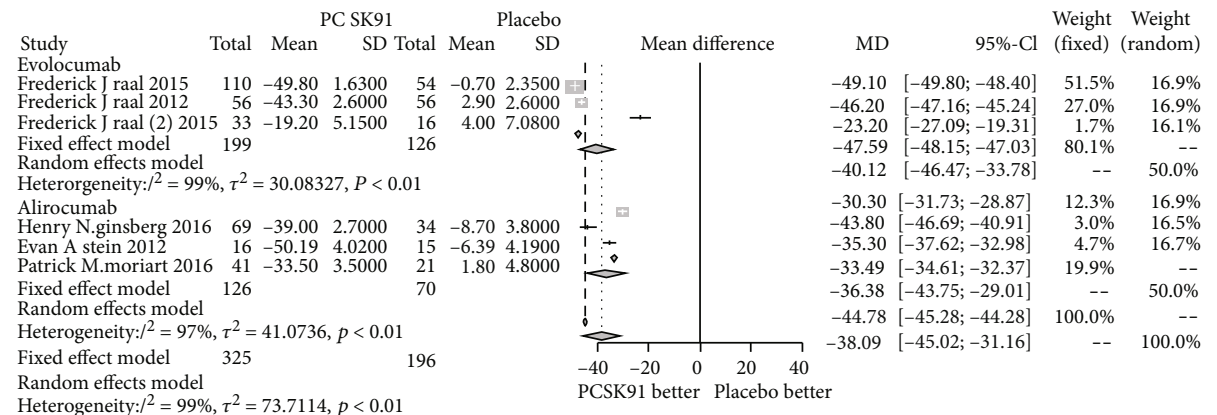

Heterogeneity: $/^{2}=99 \%, \tau^{2}=73.7114, p<0.0$

(d)

FIgUre 4: Continued. 


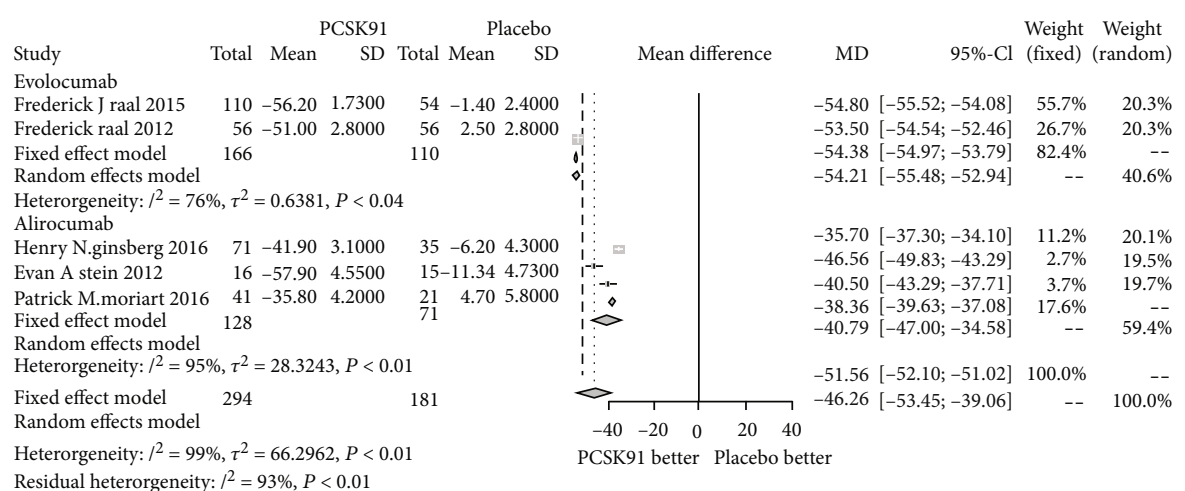

(e)

PC SK91 Placebo

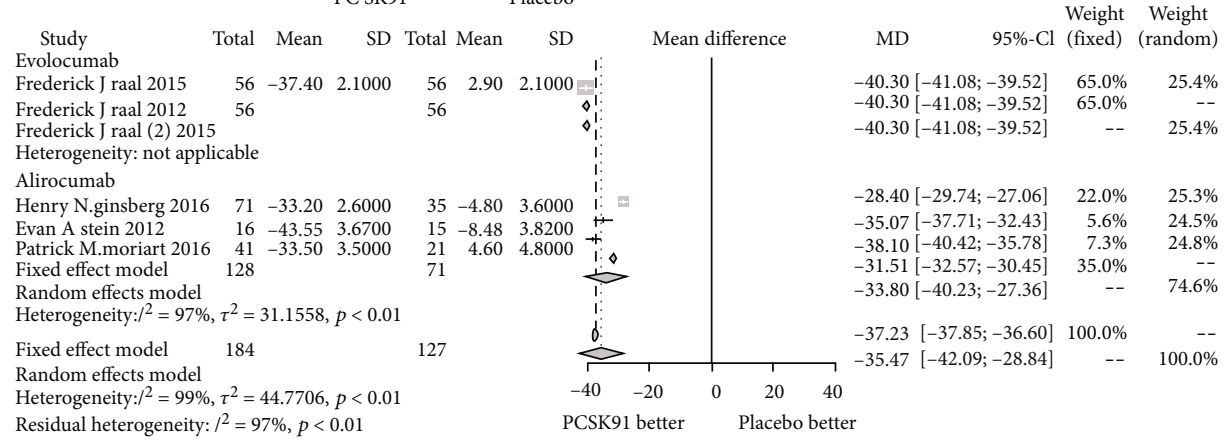

(f)

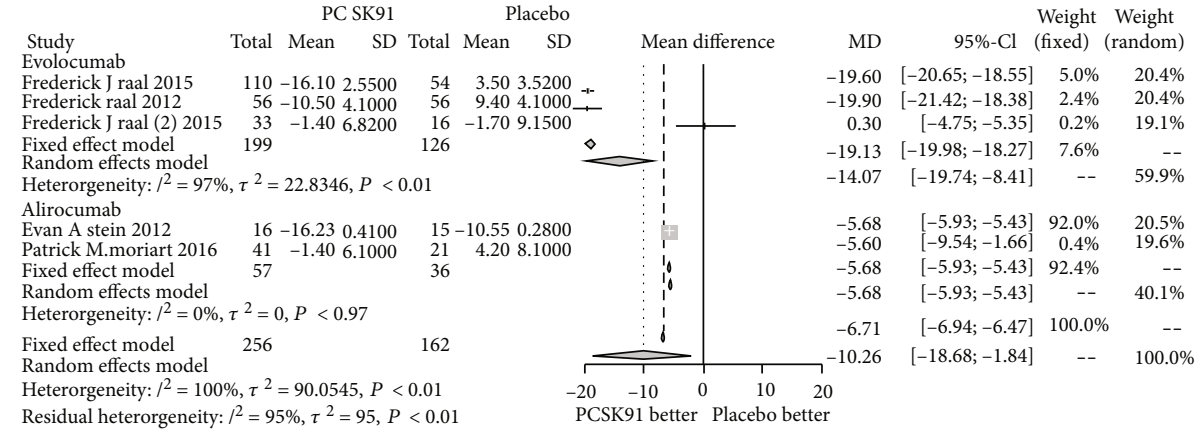

(g)

\begin{tabular}{|c|c|c|c|c|c|c|c|c|c|c|c|}
\hline \multirow[b]{2}{*}{ Study } & \multicolumn{4}{|c|}{ PC SK91 } & \multicolumn{2}{|r|}{ Placebo } & \multirow[b]{2}{*}{ Mean difference } & \multirow[b]{2}{*}{$\mathrm{MD}$} & \multirow[b]{2}{*}{$95 \%-\mathrm{Cl}$} & \multirow{2}{*}{$\begin{array}{l}\text { Weight } \\
\text { (fixed) }\end{array}$} & \multirow{2}{*}{$\begin{array}{c}\text { Weight } \\
\text { (random) }\end{array}$} \\
\hline & Total & Mean & SD & Total & Mean & SD & & & & & \\
\hline $\begin{array}{l}\text { Evolocumab } \\
\text { Frederick J raal } 2015 \\
\text { Frederick J raal } 2012 \\
\text { Frederick J raal (2) } 2015 \\
\text { Fixed effect model } \\
\text { Random effects model } \\
\text { Heterorgeneity: } /^{2}=99 \%\end{array}$ & $\begin{array}{r}110 \\
56 \\
15 \quad 33 \\
199 \\
\%, \tau^{2}=\end{array}$ & $\begin{array}{r}-22.90 \\
-19.10 \\
-9.40 \\
\\
=45.3409\end{array}$ & $\begin{array}{r}2.3500 \\
3.1000 \\
6.0800 \\
\\
9, P<0.0\end{array}$ & $\begin{array}{r}54 \\
56 \\
16 \\
126 \\
\\
01\end{array}$ & $\begin{array}{l}8.70 \\
4.10 \\
2.40\end{array}$ & $\begin{array}{l}3.3200 \\
3.1000 \\
8.1900\end{array}$ & & $\begin{array}{l}-31.60 \\
-23.20 \\
-11.80 \\
-27.59 \\
-27.56\end{array}$ & $\begin{array}{r}{[-32.59 ;-30.61]} \\
{[-24.35 ;-22.05]} \\
{[-16.32 ;-7.28]} \\
{[-28.33 ;-26.85]} \\
{[-30.33 ;-14.78]}\end{array}$ & $\begin{array}{r}45.0 \% \\
33.4 \% \\
2.2 \% \\
80.6 \% \\
\text { ] }] \\
\text { ] }--\end{array}$ & $\begin{array}{c}17.1 \% \\
17.1 \% \\
16.0 \% \\
-- \\
50.2 \%\end{array}$ \\
\hline $\begin{array}{l}\text { Alirocumab } \\
\text { Henry N.ginsberg } 2016 \\
\text { Evan A stein } 2012 \\
\text { Patrick M.moriart } 2016 \\
\text { Fixed effect model } \\
\text { Random effects model } \\
\text { Heterogeneity: } /^{2}=94 \% \text {, }\end{array}$ & 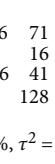 & $\begin{array}{r}-23.50 \\
-23.38 \\
-5.00\end{array}$ & $\begin{array}{l}3.7000 \\
6.2200 \\
5.9000\end{array}$ & $\begin{array}{l}35 \\
15 \\
21 \\
71\end{array}$ & $\begin{array}{l}-8.70 \\
-3.91 \\
-0.90\end{array}$ & $\begin{array}{l}5.0000 \\
2.9000 \\
8.0000\end{array}$ & ${ }_{\circ}^{-}$ & $\begin{array}{r}-14.80 \\
-19.47 \\
-4.10 \\
-14.10 \\
-12.89 \\
-24.97\end{array}$ & $\begin{array}{r}{[-16.67 ;-12.93]} \\
{[-22.85 ;-16.09]} \\
{[-7.97 ;-0.23]} \\
{[-15.61 ;-12.60]} \\
{[-20.17 ;-5.61]} \\
{[-25.64 ;-24.31]}\end{array}$ & $\begin{array}{r}12.6 \% \\
3.8 \% \\
2.9 \% \\
19.4 \% \\
-- \\
] \\
] \\
]\end{array}$ & $\begin{array}{r}17.0 \% \\
16.5 \% \\
16.3 \% \\
--- \\
49.8 \% \\
--\end{array}$ \\
\hline $\begin{array}{l}\text { Fixed effect model } \\
\text { Random effects model } \\
\text { Heterogeneity: } /^{2}=98 \%\end{array}$ & $\begin{array}{r}327 \\
\%, \tau^{2}=\end{array}$ & 76.5447 & & 197 & & & $\begin{array}{cccc}0 & -10 & 0 & 10 \\
1 & \text { better } & \text { Placebo }\end{array}$ & -17.65 & {$[-24.75 ;-10.55]$} & -- & $100.0 \%$ \\
\hline
\end{tabular}

(h)

FIGURE 4: Forest plots depicting the effect of PCSK9 monoclonal antibody on FH; (a) on LDL-C; (b) on HDL-C; (c) on Apo-A1; (d) on ApoB; (e) on non-HDL-C; (f) on TC; (g) on TG; (h) on Lp(a). 


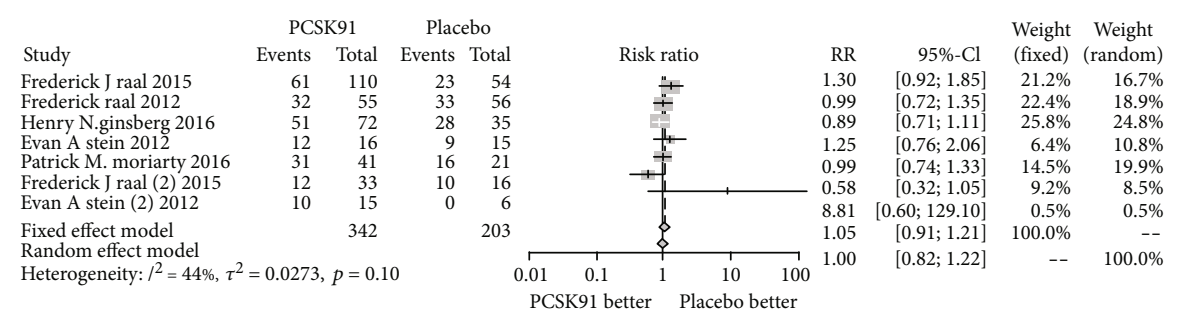

(a)

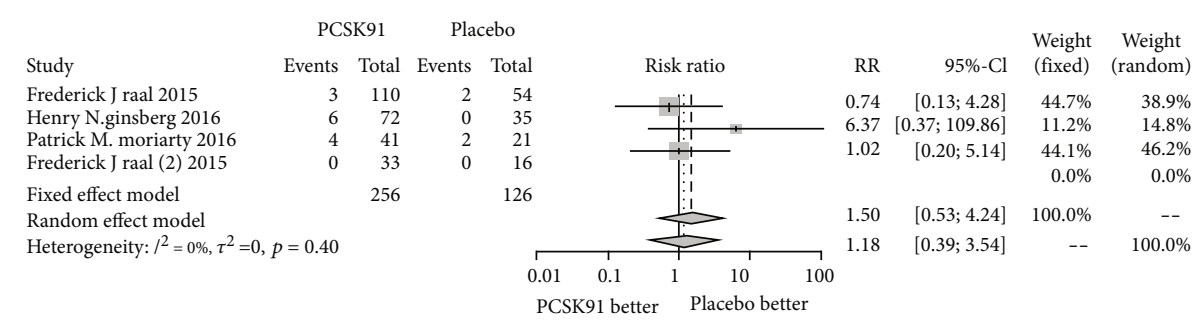

(b)

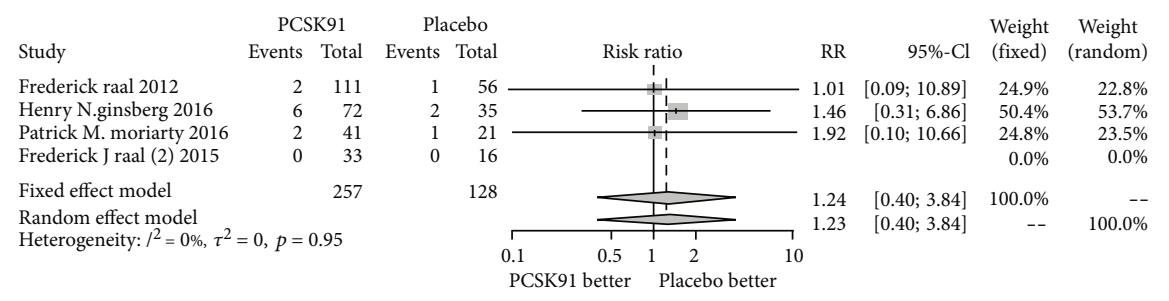

(c)

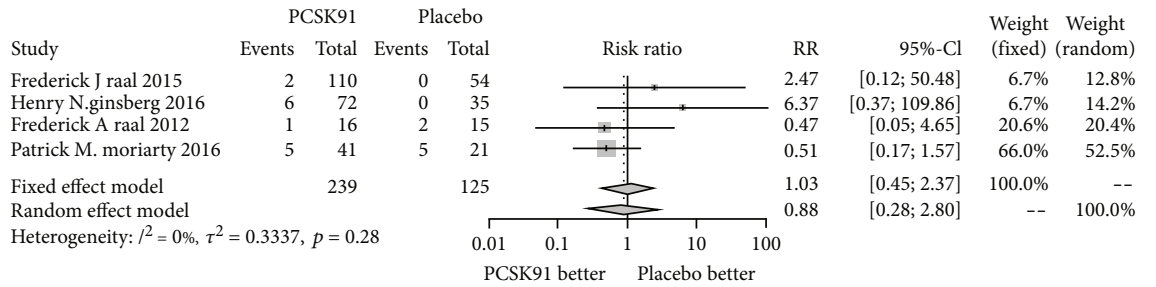

(d)

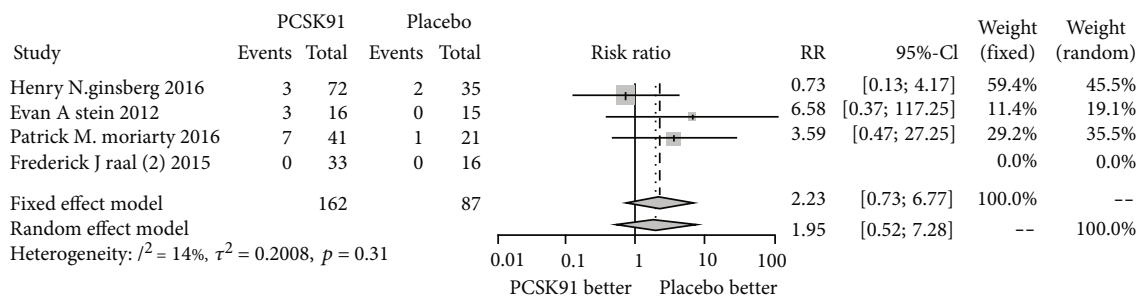

(e)

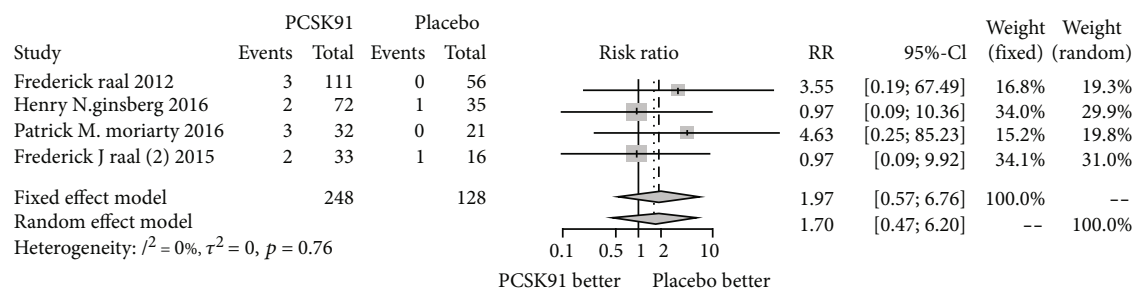

(f)

Figure 5: Continued. 


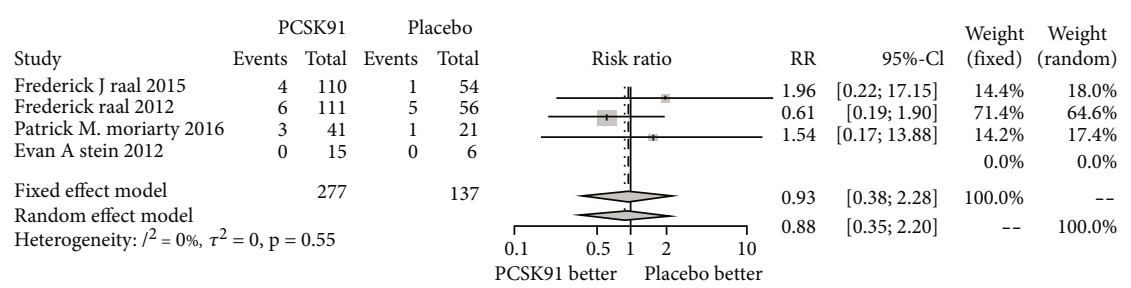

(g)

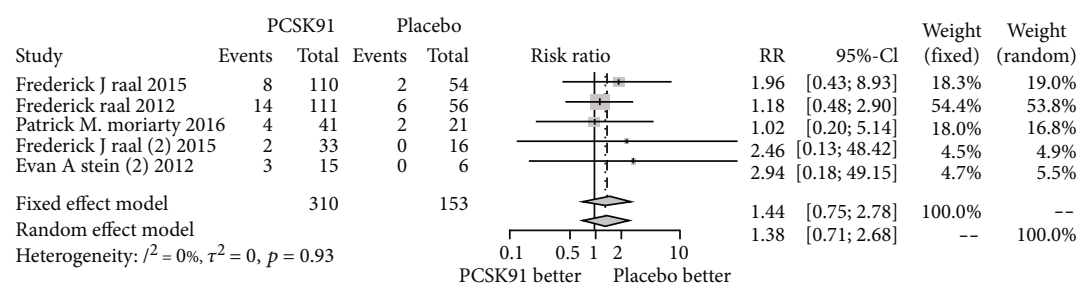

(h)

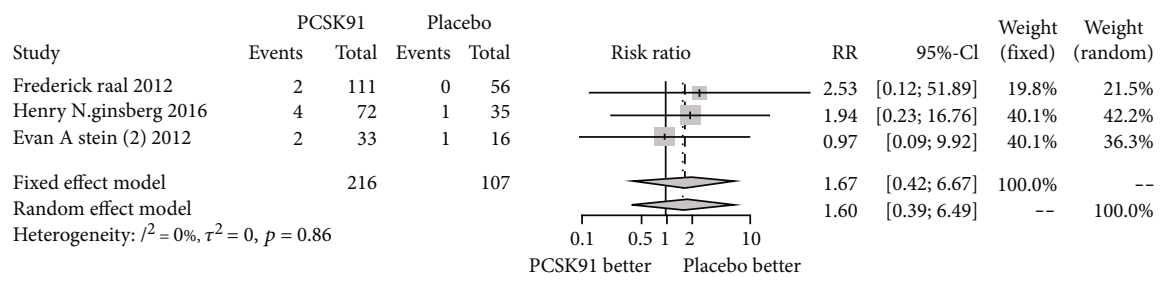

(i)

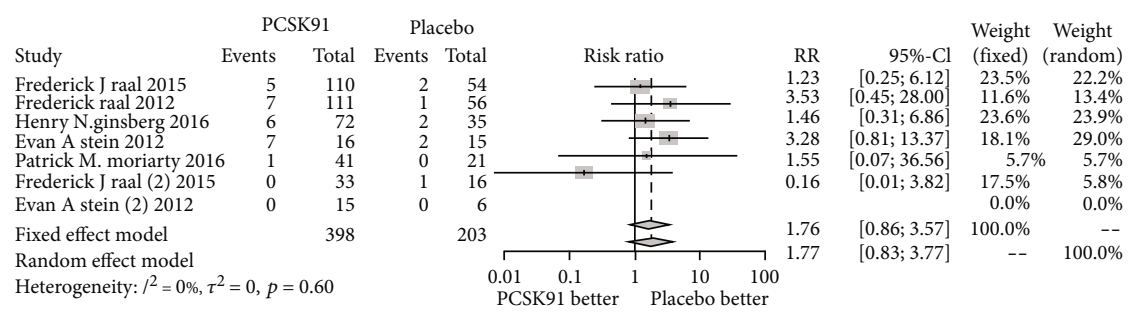

(j)

FIGURE 5: Forest plot depicting the adverse event rates of PCSK9 monoclonal antibody on FH compared with placebo controls on adverse events, serious events, and laboratory adverse events: (a) any adverse events; (b) serious adverse events; (c) leading to treatment discontinuation; (d) adjudicated cardiovascular events; (e) nervous system disorders; (f) creatine kinase $(\mathrm{CK} \geq 3 \times \mathrm{ULN})$; (g) headache; (h) nasopharyngitis; (i) abnormal liver function risk (AST/ALT $\geq 3 \times \mathrm{ULN}$ ); (j) injection site reactions.

Table 2: Prespecified safety end points. No statistical differences between the PCSK9-mAbs and control groups.

\begin{tabular}{|c|c|c|c|c|c|c|}
\hline \multirow{2}{*}{ Pre-specified Safety endpoints } & \multicolumn{2}{|c|}{ PCSK9-mAbs } & \multicolumn{2}{|l|}{ Control } & \multirow{2}{*}{$\chi^{2}$} & \multirow{2}{*}{$p$ value } \\
\hline & No. of patients/objects & Rate (\%) & No. of patients/objects & Rate (\%) & & \\
\hline Any adverse events & $209 / 342$ & 0.6111111 & $119 / 203$ & 0.5862069 & 0.33 & 0.314 \\
\hline Serious adverse events & $13 / 256$ & 0.0507813 & $4 / 126$ & 0.031746 & 0.72 & 0.287 \\
\hline Nervous system disorders & $13 / 162$ & 0.0802469 & $3 / 87$ & 0.0344828 & 1.972 & 0.127 \\
\hline Injection site reactions & $26 / 398$ & 0.0653266 & $8 / 203$ & 0.0394089 & 1.692 & 0.131 \\
\hline Leading to treatment discontinuation & $10 / 257$ & 0.0446429 & $4 / 128$ & 0.03125 & 0.143 & 0.477 \\
\hline Nasopharyngitis & $31 / 310$ & 0.1 & $10 / 153$ & 0.0653595 & 1.523 & 0.144 \\
\hline Back pain & $7 / 151$ & 0.0463576 & $2 / 75$ & 0.0266667 & 0.508 & 0.377 \\
\hline Headache & $13 / 277$ & 0.0469314 & $7 / 137$ & 0.0510949 & 0.035 & 0.513 \\
\hline ALT, AST, or both $\geq 3 \times$ ULN & $8 / 216$ & 0.037037 & $2 / 107$ & 0.0186916 & 0.803 & 0.3 \\
\hline Positively adjudicated cardiovascular events & $14 / 239$ & 0.0585774 & $7 / 125$ & 0.056 & 0.01 & 0.563 \\
\hline Creatinine kinase $\geq 3 \times \mathrm{ULN}$ & $10 / 248$ & 0.0403226 & $2 / 128$ & 0.0169492 & 1.667 & 0.164 \\
\hline
\end{tabular}


Study

Omitting frederick J raal 2015

Omitting frederick raal 2012

Omitting henry N.ginsberg 2016

Omitting evan A stein 2012

Omitting patrick M.moriarty 2016

Omitting frederick J raal (2) 2015

Omitting evan A stein (2) 2012

Fixed effect model

Study

Omitting frederick J raal 2015

Omitting frederick raal 2012

Omitting henry N.ginsberg 2016

Omitting evan A stein 2012

Omitting patrick M.moriarty 2016

Omitting frederick J raal (2) 2015

Fixed effect model

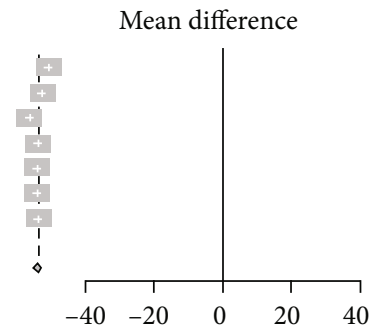

MD

95\%-Cl

(a)

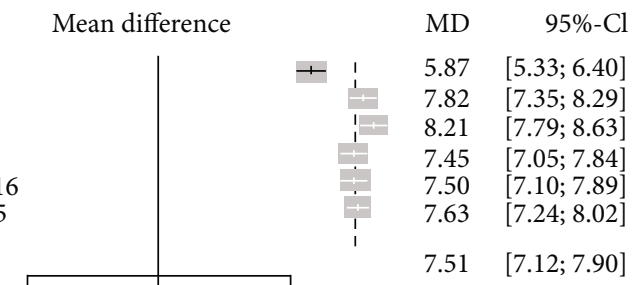

(b)

Study

Omitting frederick J raal 2015

Omitting frederick raal 2012

Omitting evan A stein 2012

Omitting patrick M.moriarty 2016

Fixed effect model

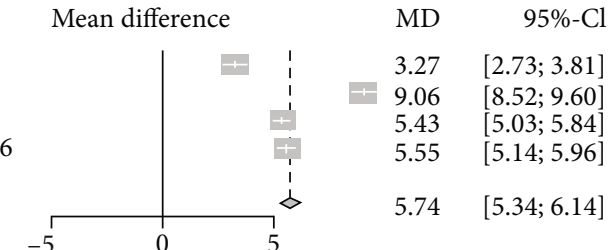

(c)

Study

Omitting frederick J raal 2015

Omitting frederick raal 2012

Omitting henry N.ginsberg 2016

Omitting evan A stein 2012

Omitting patrick M.moriarty 2016

Omitting frederick J raal (2) 2015

Fixed effect model

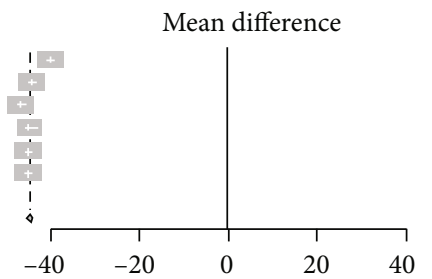

MD

$95 \%-\mathrm{Cl}$

$-40.20 \quad[-40.92 ;-39.48$

$-44.26 \quad[-44.84 ;-43.67$

$-46.81 \quad[-47.34 ;-46.27]$

$-44.81 \quad[-45.32 ;-44.30]$

$-45.25 \quad[-45.76 ;-44.73]$

$-45.14 \quad[-45.65 ;-44.64]$

$-44.78 \quad[-45.28 ;-44.28]$

(d)

Study

Omitting frederick J raal 2015

Omitting frederick raal 2012

Omitting henry N.ginsberg 2016

Omitting evan A stein 2012

Omitting patrick M.moriarty 2016

Fixed effect model

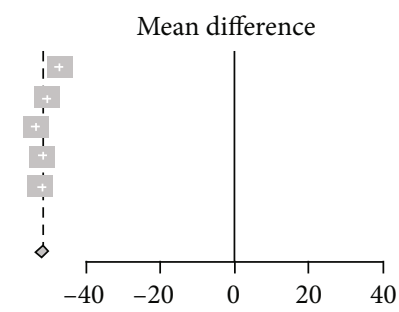

MD $\quad 95 \%-C l$

$-47.48 \quad[-48.28 ;-46.67]$

$-50.85 \quad[-51.48 ;-50.23]$

$-53.57 \quad[-54.14 ;-53.00]$

$-51.70 \quad[-52.24 ;-51.15]$

$-51.98 \quad[-52.53 ;-51.44]$

$-51.56 \quad[-52.10 ;-51.02]$

(e)

Study

Omitting frederick J raal 2015

Omitting henry N.ginsberg 2016

Omitting evan A stein 2012

Omitting patrick M.moriarty 2016

Fixed effect model

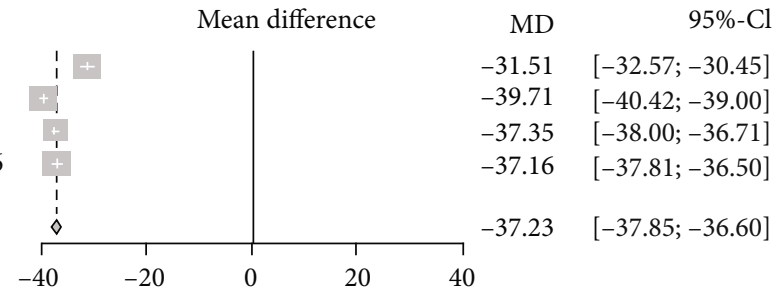

(f)

Figure 6: Continued. 
Study

Omitting frederick J raal 2015

Omitting frederick raal 2012

Omitting evan A stein 2012

Omitting frederick J raal (2) 2015

Fixed effect model
Omitting patrick M.moriarty 2016

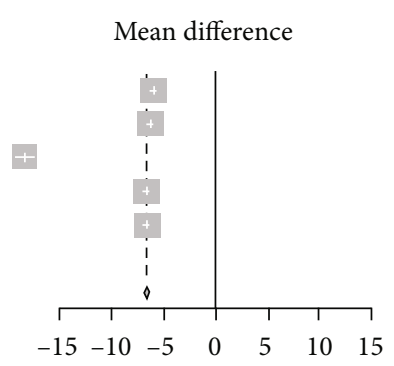

(g)

Study

Omitting frederick J raal 2015

Omitting frederick raal 2012

Omitting henry N.ginsberg 2016

Omitting evan A stein 2012

Omitting patrick M.moriarty 2016

Omitting frederick J raal (2) 2015

Fixed effect model

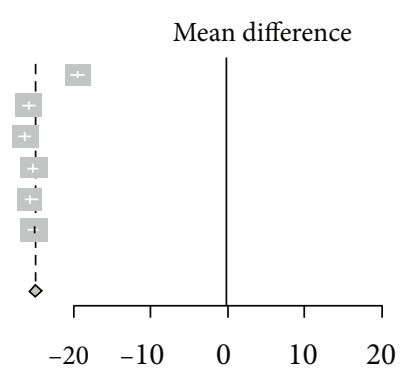

(h)

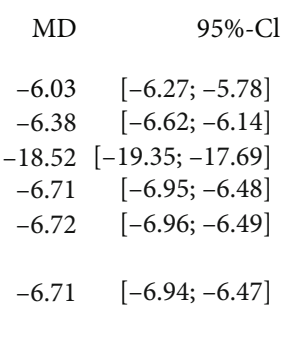

$$
\begin{array}{rr}
\text { MD } & 95 \%-\mathrm{Cl} \\
-19.54 & {[-20.43 ;-18.64]} \\
-25.86 & {[-26.67 ;-25.05]} \\
-26.44 & {[-27.15 ;-25.73]} \\
-25.19 & {[-25.87 ;-24.52]} \\
-25.60 & {[-26.28 ;-24.93]} \\
-25.26 & {[-25.93 ;-24.59]} \\
-24.97 & {[-25.64 ;-24.31]}
\end{array}
$$

FIgure 6: Sensitivity analysis of efficacy outcomes: (a) on LDL-C; (b) on HDL-C; (c) on Apo-A1; (d) on Apo-B; (e) on non-HDL-C; (f) on TC; (g) on TG; (h) on $\operatorname{Lp}(\mathrm{a})$.

incidence of common adverse events (RR: $1.00,95 \%$ CI: 0.82 to $1.22, I^{2}: 44 \%, p=0.10$ ), serious adverse events (RR: $1.18,95 \%$ CI: 0.39 to $\left.3.54, I^{2}: 0 \%, p=0.40\right)$, and leading to treatment discontinuation (RR: 1.23 , 95\% CI: 0.40 to $3.84, I^{2}: 0 \%, p=0.95$ ) implied no obvious differences versus placebo. No significant heterogeneity was found in positively adjudicated cardiovascular events by RR: $0.88,95 \%$ CI: 0.28 to $2.80, I^{2}: 22 \%, p=0.28$; nervous system disorders by RR: $1.95,95 \% \mathrm{CI}: 0.52$ to $7.28, I^{2}$ : $14 \%, p=0.31$; creatine kinase $(\mathrm{CK} \geq 3 \times \mathrm{ULN})$ by RR: 1.70 , 95\% CI: 0.47 to $6.20, I^{2}: 0 \%, p=0.76$; headache by RR: 0.88 , $95 \%$ CI: 0.35 to $2.20, I^{2}: 0 \%, p=0.55$; nasopharyngitis by RR: $1.38,95 \%$ CI: 0.71 to $2.68, I^{2}: 0 \%, p=0.93$; abnormal liver function risk (AST/ALT $\geq 3 \times \mathrm{ULN}$ ) in patients by RR: $1.60,95 \%$ CI: 0.39 to $6.49, I^{2}: 0 \%, p=0.86$; and injection site reactions by RR: $1.77,95 \%$ CI: 0.83 to $3.77, I^{2}: 0 \%, p=0.60$, versus placebo (Figure 5).

Moreover, an additional table that describes the safety events of interest, common adverse events, and laboratory adverse events of PCSK9-mAbs was included, and we found no significant differences between the PCSK9-mAbs and control groups. A chi-square $\left(\chi^{2}\right)$ statistic was used to assess the magnitude of heterogeneity, and a $p$ value $<0.05$ was considered to be statistically significant (Table 2).

3.5. Sensitivity Analysis. To explain the high heterogeneity observed among all efficacy outcomes, we performed leaveone-out sensitivity analysis among the studies. We found that the statistical significance or nonsignificance of the differences between groups was not altered. This suggested that none of the included studies individually changed the overall result (Figure 6). Moreover, there was also no change in safety outcomes (Figure 7).

\section{Discussion}

To the best of our knowledge, this is the first meta-analysis using sufficient clinical outcomes to systematically analyze the efficacy and safety of PCSK9-mAbs in the treatment of $\mathrm{FH}$ patients. In the present analysis, a total of 7 studies encompassing 926 patients with FH were included. The main aim is to solve whether PCSK9-mAbs treatment can reduce the levels of lipids of $\mathrm{FH}$ patients with satisfactory safety and tolerability.

$\mathrm{FH}$ is an inherited disease due to a genetic mutation and is not caused by the external environment or improper lifestyle. As mentioned before, FH includes two main subtypes: $\mathrm{HeFH}$ and homozygous FH. They are different in symptoms, risks, and treatments. In genetics, $\mathrm{HeFH}$ is caused by a pathogenic variant in one allele, while biallelic mutations in one of the known genes or compound heterozygosity for two different mutations in the same or different candidate genes cause homozygous $\mathrm{FH}$. HeFH affects between one in 250 and one in 300 people worldwide, and the prevalence of homozygous FH may be 1 in 160,000 [20]. The risk of premature CHD in heterozygous $\mathrm{FH}$ is elevated approximately 20-fold [4], and homozygous $\mathrm{FH}$ patients develop CHD early by the second decade of life. In homozygous FH, valvular and supravalvular aortic stenosis induced by lipid deposition has also been reported, whereas rarely in HeFH [21]. To date, 12 meta-analysis studies have analyzed the efficacy and safety of PCSK9-mAbs in hypercholesterolemia [9, 22-32]. Among these studies, there were two in $\mathrm{FH}$ patients. However, one report studied the role and safety of evolocumab but did not include alirocumab [33]. In another study, although the role and safety of PCSK9-mAbs, including evolocumab and alirocumab, were discussed, the patients with 


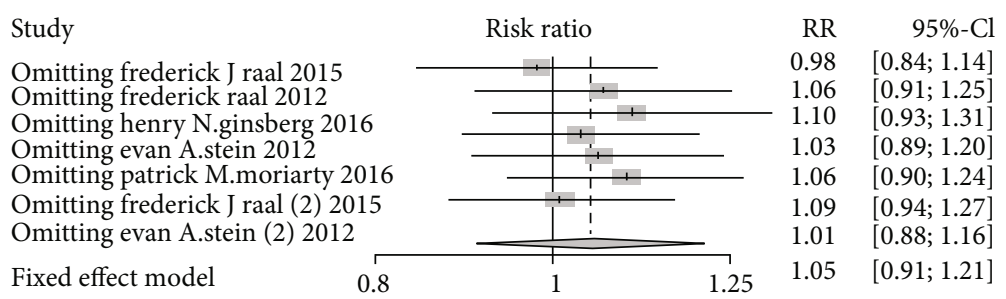

(a)

Study

Omitting frederick J raal 2015 Omitting henry N.ginsberg 2016

Omitting patrick M.moriarty 2016 Omitting frederick J raal (2) 2015

Fixed effect model

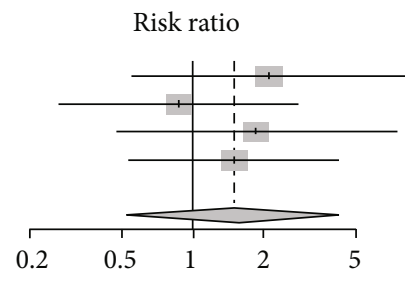

RR

$95 \%-\mathrm{Cl}$

$2.11 \quad[0.55 ; 8.11]$

$0.88 \quad[0.27 ; 2.88]$

$1.87 \quad[0.47 ; 7.40]$

$1.50 \quad[0.53 ; 4.24]$

$1.50 \quad[0.53 ; 4.24]$

(b)

Study

Omitting frederick raal 2012

Omitting henry N.ginsberg 2016

Omitting patrick M.moriarty 2016

Omitting frederick J raal (2) 2015

Fixed effect model

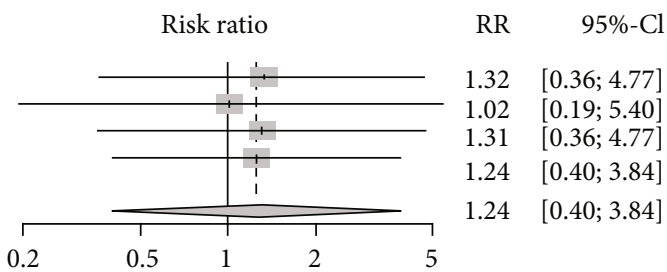

(c)

Study

Omitting frederick J raal 2015

Omitting henry N.ginsberg 2016

Omitting evan A.stein 2012

Omitting patrick M.moriarty 2016

Fixed effect model

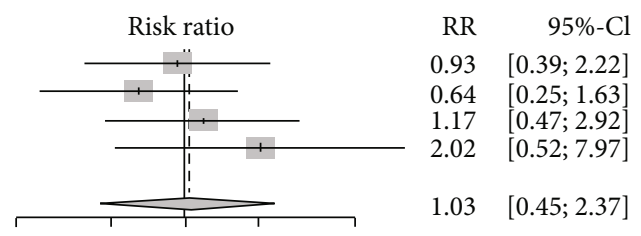

(d)

Study

Omitting henry N.ginsberg 2016 Omitting evan A.stein 2012

Omitting patrick M.moriarty 2016

Omitting frederick J raal (2) 2015

Fixed effect model

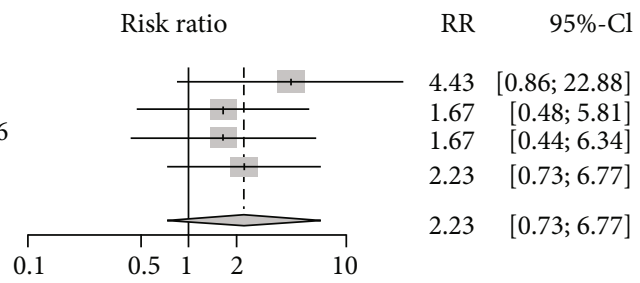

(e)

Study

Omitting frederick J raal 2012 Omitting henry N.ginsberg 2016

Omitting patrick M.moriarty 2016

Omitting frederick J raal (2) 2015

Fixed effect model

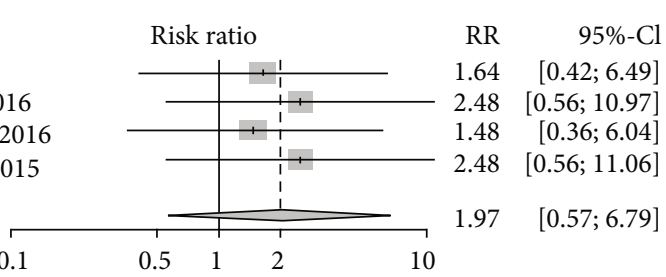

(f)

Figure 7: Continued. 


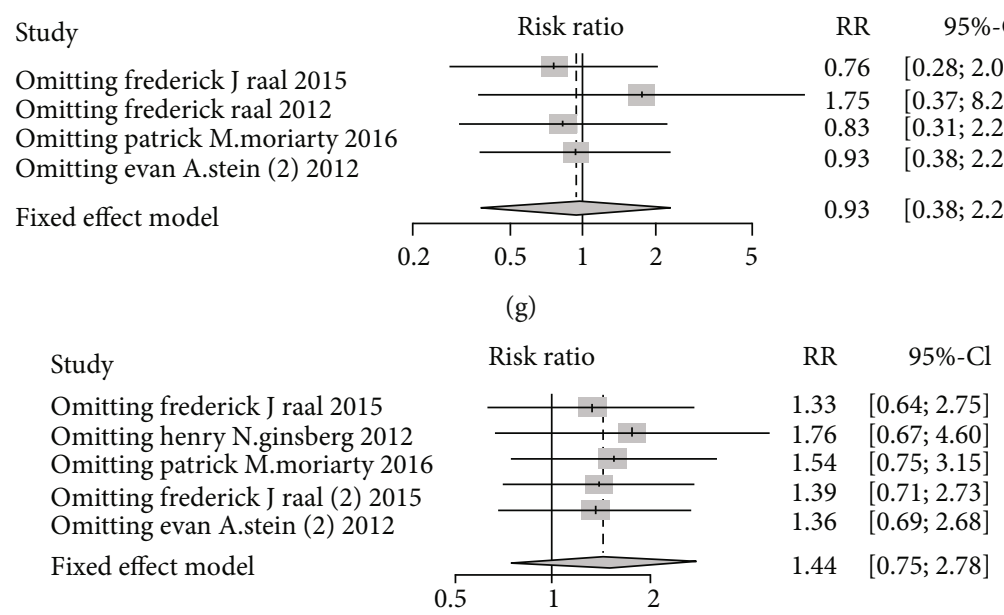

(h)

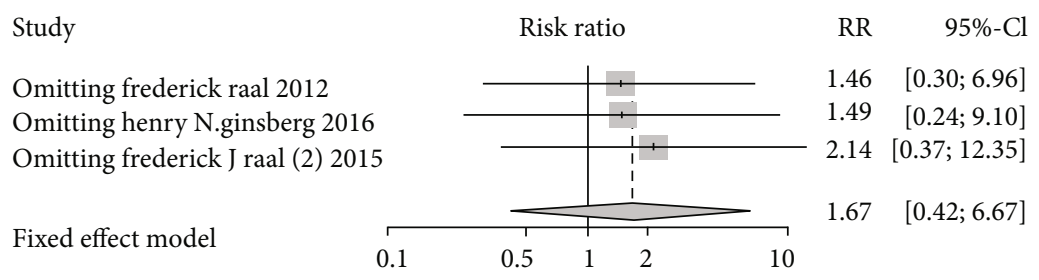

(i)

Study
Omitting frederick J raal 2015
Omitting frederick raal 2012
Omitting henry N.ginsberg 2016
Omitting evan A.stein 2012
Omitting patrick M.moriarty 2016
Omitting frederick J raal (2) 2015
Omitting evan A.stein (2) 2012
Fixed effect model

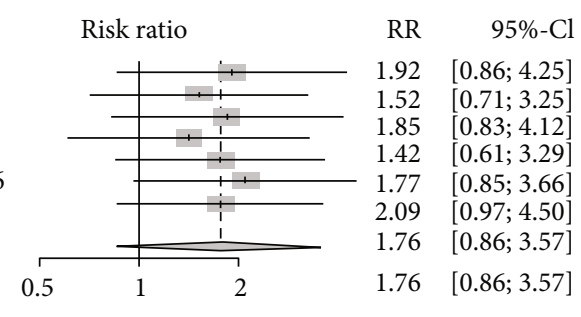

(j)

FIGURE 7: Sensitivity analysis of safety outcomes: (a) any adverse events; (b) serious adverse events; (c) leading to treatment discontinuation; (d) adjudicated cardiovascular events; (e) nervous system disorders; ( $\mathrm{f}$ ) creatine kinase (CK $\geq 3 \times \mathrm{ULN}$ ); (g) headache; (h) nasopharyngitis; (i) abnormal liver function risk (AST/ALT $\geq 3 \times U L N)$; (j) injection site reactions.

FH were controversial because the data extracted by the authors did not exclude the non-FH patients included in the clinical study [10]. Therefore, strictly speaking, our meta-analysis is the first study to systematically analyze the efficacy and safety of PCSK9-mAbs in the treatment of $\mathrm{FH}$ patients alone. In addition, we conducted a variety of sensitivity analyses for the included literature to ensure the reliability of the literature screening and results, including leave-one-out sensitivity analysis, contour-enhanced funnel plots, and the Harbord modification of Egger test. Moreover, we conducted a subgroup analysis of PCSK9-mAbs and analyzed the efficacy outcomes and safety outcomes of evolocu$\mathrm{mab}$ and alirocumab in the treatment of $\mathrm{FH}$, respectively.

In this study, we conducted a systematic evaluation of the efficacy and safety of PCSK9-mAbs in FH patients. The results of this study showed that PCSK9-mAbs reduced the level of the main research index LDL-C and also significantly reduced the levels of TG, TC, non-HDL-C, and Apo B, Lp(a) and increased the levels of HDL-C and Apo-A1, which are beneficial lipopro- teins. Elevated LDL is an important pathological factor for CVD. The latest Mendelian Randomization Study Tips published by JACC based on the UK Biobank and Global Lipid Genetics Consortium (Global Lipid Genetics Consortium) demonstrated that LDL cholesterol and triglycerides induced myocardial remodeling by increasing LV mass, suggesting that they influence the development of CVD not only through atherosclerosis but also by causing adverse alterations in cardiac structure and function $[34,35]$. In the present study, we showed that PCSK9-mAbs significantly reduced LDL and TG levels by $-49.14 \%$ and $-10.26 \%$, respectively. The Apo-B/ApoA1 ratio has been previously suggested to be a better risk indicator for CVD and MI than the level of lipids [36], and we demonstrated that in the PCSK9-mAbs treatment group, the level of Apo-B decreased while that of Apo-A1 increased. This result indicates that PCSK9-mAbs therapy can greatly lower the primary risk factors for heart disease with an obvious decrease in the Apo-B/Apo-A1 ratio. Recent studies have reported that $\mathrm{Lp}(\mathrm{a})$ not only serves as a "traditional" 
atherosclerotic cardiovascular disease (ASCVD) risk factor but also improves the accuracy of cardiovascular risk stratification [37]. Compelling evidence from traditional epidemiological, genome-wide association, and Mendelian randomization studies has revealed that elevated plasma $\mathrm{Lp}$ (a) level increases the risk of acute myocardial infarction (AMI), ischemic stroke, calcific aortic valve disease, and peripheral arterial disease in non-FH patients [38]. Elevated lipoprotein (a) was found to be a significant CVD risk factor in $\mathrm{HeFH}$ [39], and Lp(a) level above $50 \mathrm{mg} / \mathrm{dL}$ is recently found to be an independent risk factor for calcific aortic valvulopathy among $\mathrm{HeFH}$ patients [40]. Safety analysis demonstrated that PCSK9-mAbs showed good safety, and the incidence of common and serious adverse reactions was basically the same as that of the placebo group in addition to abnormal liver function risk.

Several limitations should be taken into consideration. First, significant heterogeneities were observed in most of the efficacy outcomes, which may be related to the patient's baseline level, drug intervention time, the type and dose of PCSK9mAbs, etc., but we failed to reveal the heterogeneities by dividing into subgroups or using sensitivity methods. Second, there are still a number of large-scale randomized clinical controlled studies in progress, and we should take caution in interpreting the results of the meta-analysis when combining heterogeneous data sets. Third, most of the treatment cycles included in clinical studies were between 12 and 24 weeks, and the adverse reactions that require long-term observation could not be effectively evaluated. Despite these limitations, our meta-analysis proves that PCSK9-mAbs exert significant protection from $\mathrm{FH}$, including decreasing the plasma levels of LDL-C and Lp(a), TC, TG, and Apo-B and increasing the plasma levels of HDL-C and Apo-A1. Outcomes are sufficient enough to compensate our clinical guidelines. Hopefully, its long-term therapeutic efficacy, safety, and clinical outcomes should be confirmed by more RCTs.

\section{Conclusion}

In this review, we presented evidence from 7 published clinical trials and suggested that among $926 \mathrm{FH}$ patients, PCSK9-mAbs significantly decreased the level of LDL-C and other lipids with satisfactory safety and tolerability.

\section{Conflicts of Interest}

The authors have no conflicts of interest to disclose.

\section{Acknowledgments}

This work was supported by grants from the National Natural Science Foundation of China (No. 82000062, 81960015 to WF. Zhang, and 81800051 to TT. Zhu), the Young Talents Project Foundation from Jiangxi Provincial Department of Science and Technology (20204BCJ23020), the Science Foundation for Distinguished Young Scholars of Jiangxi Province (20212ACB216008), National College Students Innovation Training Program of Henan Province (202010472010), and Research Foundation of Xinxiang Medical University (XYBSKYZZ201812).

\section{References}

[1] A. Onorato and A. C. Sturm, "Heterozygous familial hypercholesterolemia," Circulation, vol. 133, no. 14, pp. e587-e589, 2016.

[2] A. Thedrez, D. J. Blom, S. Ramin-Mangata et al., "Homozygous familial hypercholesterolemia patients with identical mutations variably express the LDLR (low-density lipoprotein receptor): implications for the efficacy of evolocumab," Arteriosclerosis, Thrombosis, and Vascular Biology, vol. 38, no. 3, pp. 592-598, 2018.

[3] C. C. Huang and M. J. Charng, "Genetic diagnosis of familial hypercholesterolemia in asia," Frontiers in Genetics, vol. 11, 2020.

[4] G. F. Watts, B. Lewis, and D. R. Sullivan, "Familial hypercholesterolemia: a missed opportunity in preventive medicine," Nature Clinical Practice. Cardiovascular Medicine, vol. 4, no. 8, pp. 404-405, 2007.

[5] B. G. Nordestgaard, M. J. Chapman, S. E. Humphries et al., "Familial hypercholesterolaemia is underdiagnosed and undertreated in the general population: guidance for clinicians to prevent coronary heart disease: consensus statement of the European Atherosclerosis Society," European Heart Journal, vol. 34, no. 45, pp. 3478-3490, 2013.

[6] V. Blanchard, I. Khantalin, S. Ramin-Mangata, K. Chémello, B. Nativel, and G. Lambert, "PCSK9: from biology to clinical applications,” Pathology, vol. 51, no. 2, pp. 177-183, 2019.

[7] M. Hilvo, H. Simolin, J. Metso et al., "PCSK9 inhibition alters the lipidome of plasma and lipoprotein fractions," Atherosclerosis, vol. 269, pp. 159-165, 2018.

[8] J. J. P. Kastelein, S. E. Nissen, D. J. Rader et al., "Safety and efficacy of LY3015014, a monoclonal antibody to proprotein convertase subtilisin/kexin type 9 (PCSK9): a randomized, placebo-controlled phase 2 study," European Heart Journal, vol. 37, no. 17, pp. 1360-1369, 2016.

[9] P. M. Ridker, L. M. Rose, J. J. P. Kastelein et al., "Cardiovascular event reduction with PCSK9 inhibition among 1578 patients with familial hypercholesterolemia: Results from the SPIRE randomized trials of bococizumab," Journal of Clinical Lipidology, vol. 12, no. 4, pp. 958-965, 2018.

[10] L. J. Qian, Y. Gao, Y. M. Zhang, M. Chu, J. Yao, and D. Xu, "Therapeutic efficacy and safety of PCSK9-monoclonal antibodies on familial hypercholesterolemia and statin-intolerant patients: a meta-analysis of 15 randomized controlled trials," Scientific Reports, vol. 7, no. 1, p. 238, 2017.

[11] X. Y. Ge, T. T. Zhu, X. Y. Zhang, Y. Liu, Y. Wang, and W. Zhang, "Gender differences in pulmonary arterial hypertension patients with BMPR2 mutation: a meta-analysis," Respiratory Research, vol. 21, no. 1, p. 44, 2020.

[12] J. P. Higgins, J. Thomas, J. Chandler et al., "Cochrane handbook for systematic reviews of interventions version 6.0 (updated July 2019)," in Cochrane, John Wiley \& Sons, 2019, http://training.cochrane.org/handbook.

[13] P. M. Moriarty, K. G. Parhofer, S. P. Babirak et al., "Alirocumab in patients with heterozygous familial hypercholesterolaemia undergoing lipoprotein apheresis: the ODYSSEY ESCAPE trial," European Heart Journal, vol. 37, no. 48, pp. 3588-3595, 2016.

[14] E. A. Stein, D. Gipe, J. Bergeron et al., "Effect of a monoclonal antibody to PCSK9, REGN727/SAR236553, to reduce lowdensity lipoprotein cholesterol in patients with heterozygous familial hypercholesterolaemia on stable statin dose with or 
without ezetimibe therapy: a phase 2 randomised controlled trial," Lancet, vol. 380, no. 9836, pp. 29-36, 2012.

[15] H. N. Ginsberg, D. J. Rader, F. J. Raal et al., "Efficacy and safety of alirocumab in patients with heterozygous familial hypercholesterolemia and LDL-C of $160 \mathrm{mg} / \mathrm{dl}$ or higher," Cardiovascular Drugs and Therapy, vol. 30, no. 5, pp. 473-483, 2016.

[16] F. J. Raal, E. A. Stein, R. Dufour et al., "PCSK9 inhibition with evolocumab (AMG 145) in heterozygous familial hypercholesterolaemia (RUTHERFORD-2): a randomised, double-blind, placebo- controlled trial," Lancet, vol. 385, no. 9965, pp. 331340, 2015.

[17] F. Raal, R. Scott, R. Somaratne et al., "Low-density lipoprotein cholesterol-lowering effects of AMG 145, a monoclonal antibody to proprotein convertase subtilisin/kexin type 9 serine protease in patients with heterozygous familial hypercholesterolemia: the reduction of LDL-C with PCSK9 inhibition in heterozygous familial hypercholesterolemia disorder (RUTHERFORD) randomized trial," Circulation, vol. 126, no. 20, pp. 2408-2417, 2012.

[18] F. J. Raal, N. Honarpour, D. J. Blom et al., "Inhibition of PCSK9 with evolocumab in homozygous familial hypercholesterolaemia (TESLA part B): a randomised, double-blind, placebo- controlled trial," Lancet, vol. 385, no. 9965, pp. 341$350,2015$.

[19] E. A. Stein, S. Mellis, G. D. Yancopoulos et al., "Effect of a monoclonal antibody to PCSK9 on LDL cholesterol," The New England Journal of Medicine, vol. 366, no. 12, pp. 11081118, 2012.

[20] S. Singh and V. Bittner, "Familial hypercholesterolemia-epidemiology, diagnosis, and screening," Current Atherosclerosis Reports, vol. 17, no. 2, p. 482, 2015.

[21] K. Ozumi, H. Tasaki, K. Yamashita et al., "Valvular and supravalvular aortic stenosis in heterozygous familial hypercholesterolemia, a case report," Journal of Atherosclerosis and Thrombosis, vol. 12, no. 5, pp. 289-293, 2005.

[22] A. A. A. Asbeutah, S. A. Asbeutah, and M. A. Abu-Assi, "A meta-analysis of cardiovascular outcomes in patients with hypercholesterolemia treated with inclisiran," The American Journal of Cardiology, vol. 128, pp. 218-219, 2020.

[23] Z. Zhao, S. du, S. Shen et al., "Comparative efficacy and safety of lipid-lowering agents in patients with hypercholesterolemia," Medicine (Baltimore), vol. 98, no. 6, article e14400, 2019.

[24] A. Karatasakis, B. A. Danek, J. Karacsonyi et al., "Effect of PCSK9 inhibitors on clinical outcomes in patients with hypercholesterolemia: a meta-analysis of 35 randomized controlled trials," Journal of the American Heart Association, vol. 6, no. $12,2017$.

[25] X. L. Zhang, Q. Q. Zhu, L. Zhu et al., "Safety and efficacy of anti-PCSK9 antibodies: a meta-analysis of 25 randomized, controlled trials," BMC Medicine, vol. 13, no. 1, p. 123, 2015.

[26] R. Pan, Y. Li, and H. Zhou, "Efficacy and safety of different doses of alirocumab in reducing low-density lipoprotein cholesterol levels: a network meta-analysis," Die Pharmazie, vol. 74, no. 1, pp. 8-14, 2019.

[27] E. P. Navarese, M. Kołodziejczak, V. Schulze et al., "Effects of proprotein convertase subtilisin/kexin type 9 antibodies in adults with Hypercholesterolemia," Annals of Internal Medicine, vol. 163, no. 1, pp. 40-51, 2015.

[28] C. Li, L. Lin, W. Zhang et al., "Efficiency and safety of proprotein convertase subtilisin/kexin 9 monoclonal antibody on hypercholesterolemia: a meta-analysis of 20 randomized controlled trials," Journal of the American Heart Association, vol. 4, no. 6, article e001937, 2015.

[29] X. X. He, R. Zhang, P. Y. Zuo et al., "The efficacy advantage of evolocumab (AMG 145) dosed at $140 \mathrm{mg}$ every 2 weeks versus $420 \mathrm{mg}$ every 4 weeks in patients with hypercholesterolemia: evidence from a meta-analysis," European Journal of Internal Medicine, vol. 38, pp. 52-60, 2017.

[30] H. Wan, B. Gumbiner, T. S. Joh et al., "Effects of proprotein convertase subtilisin/kexin type 9 (PCSK9) inhibition with bococizumab on lipoprotein particles in hypercholesterolemic subjects," Clinical Therapeutics, vol. 39, no. 11, pp. $2243-$ 2259.e5, 2017.

[31] M. J. Lipinski, U. Benedetto, R. O. Escarcega et al., "The impact of proprotein convertase subtilisin-kexin type 9 serine protease inhibitors on lipid levels and outcomes in patients with primary hypercholesterolaemia: a network meta-analysis," European Heart Journal, vol. 37, no. 6, pp. 536-545, 2016.

[32] H. du, X. Li, N. Su et al., "Proprotein convertase subtilisin/kexin 9 inhibitors in reducing cardiovascular outcomes: a systematic review and meta-analysis," Heart, vol. 105, no. 15, p. heartjnl-2019-314763, 2019.

[33] S. M. Eslami, S. Nikfar, M. Ghasemi, and M. Abdollahi, "Does evolocumab, as a PCSK9 inhibitor, ameliorate the lipid profile in familial hypercholesterolemia patients? A meta-analysis of randomized controlled trials," Journal of Pharmacy \& Pharmaceutical Sciences, vol. 20, pp. 81-96, 2017.

[34] R. Roberts, "Cholesterol surprisingly also induces ventricular hypertrophy," Journal of the American College of Cardiology, vol. 76, no. 21, pp. 2489-2491, 2020.

[35] N. Aung, M. M. Sanghvi, S. K. Piechnik, S. Neubauer, P. B. Munroe, and S. E. Petersen, "The effect of blood lipids on the left ventricle: a mendelian randomization study," Journal of the American College of Cardiology, vol. 76, no. 21, pp. 24772488, 2020.

[36] M. J. McQueen, S. Hawken, X. Wang et al., "Lipids, lipoproteins, and apolipoproteins as risk markers of myocardial infarction in 52 countries (the INTERHEART study): a casecontrol study," The Lancet, vol. 372, no. 9634, pp. 224-233, 2008.

[37] S. Tsimikas, "A test in context: lipoprotein(a): diagnosis, prognosis, controversies, and emerging therapies," Journal of the American College of Cardiology, vol. 69, no. 6, pp. 692-711, 2017.

[38] A. Vuorio, G. F. Watts, W. J. Schneider, S. Tsimikas, and P. T. Kovanen, "Familial hypercholesterolemia and elevated lipoprotein (a): double heritable risk and new therapeutic opportunities," Journal of Internal Medicine, vol. 287, no. 1, pp. 2-18, 2020.

[39] L. E. Akioyamen, J. Genest, A. Chu, H. Inibhunu, D. T. Ko, and J. V. Tu, "Risk factors for cardiovascular disease in heterozygous familial hypercholesterolemia: a systematic review and meta-analysis," Journal of Clinical Lipidology, vol. 13, no. 1, pp. 15-30, 2019.

[40] A. Vuorio, G. F. Watts, and P. T. Kovanen, "Lipoprotein(a) as a risk factor for calcific aortic valvulopathy in heterozygous familial hypercholesterolemia," Atherosclerosis, vol. 281, pp. 25-30, 2019. 